\title{
Tecnologias de informação aplicadas à logística internacional
}

\author{
RESUMO
}

Frederico

Gonçalves

Schwartzhaupt

frederico-sg@hotmail.com

Universidade de Caxias do Sul (UCS), Caxias do Sul, RS, Brasil

Guilherme Bergmann Borges Vieira

gbbvieir@gmail.com

niversidade de Caxias do Sul (UCS), Caxias do Sul, RS, Brasil

\section{Roberto Birch Gonçalves}

rbgoncal@ucs.br

Universidade de Caxias do Sul (UCS),

Caxias do Sul, RS, Brasil

\begin{abstract}
A tecnologia de informação (TI) tem um importante papel no desenvolvimento das atividades logísticas e no desempenho dos operadores. O objetivo deste trabalho foi analisar o papel das tecnologias de informação aplicadas à logística internacional adotada pelos agentes de carga (IFFs) atuantes no Brasil. Para tanto, foi desenvolvida uma pesquisa quantitativa composta por um questionário estruturado, enviado a uma amostra de agentes internacionais de carga com atuação no mercado brasileiro com diferentes posicionamentos estratégicos e operando em diferentes setores. Os resultados evidenciam as TI mais e menos adotadas e as consideradas mais e menos importantes e, então, a relação entre essas categorias e o meio em que estão inseridas.
\end{abstract}

PALAVRAS-CHAVE: Logística. Tecnologia da Informação. Agentes Internacionais de Carga. IFFs. 


\section{INTRODUÇÃO}

No cenário global atual, a atividade logística exerce, mais do que nunca, um papel fundamental para as empresas se manterem competitivas. Segundo Porter (1989), a vantagem competitiva surge da maneira como as empresas desempenham suas atividades dentro da cadeia de valor. Nesse contexto, a utilização da logística associada à tecnologia de informação é significativa para que as empresas alcancem maior competitividade. Isso se deve à forma como a informação é processada e comunicada, o que por sua vez influencia as relações entre as empresas, bem como sua interdependência.

As possibilidades abertas pelo desenvolvimento da TI tornaram possível a integração e coordenação dos canais que coletam, transmitem e processam informações e dos canais físicos de suprimento e distribuição. Portanto, a tecnologia da informação adquire importância, invadindo todo o processo e, mais ainda, influenciando seu resultado. Esse fato permite que as organizações se preocupem, além dos seus custos, com aspectos como clientes, diferenciação de produtos, inovação e cadeia de suprimentos e informação. Moresi (2000) ressalta que a informação é um dos mais importantes recursos organizacionais, mas que o sucesso está relacionado com a forma de gestão, pois $2 / 3$ da informação que os administradores obtêm para a tomada de decisão, vem de fontes humanas (DAVENPORT, 1998). Esse cenário conduz a quinta fase do supply chain management, a qual está baseada nas seguintes características: i) integração total da cadeia de valor; e ii) parcerias duradouras, porém flexíveis e adaptáveis (ÁVILA, 2006).

Diante do exposto, o presente estudo visa analisar o papel da tecnologia da informação na gestão logística dos agentes de carga/international freight forwarders (IFFs) atuantes no Brasil. Em decorrência, a questão que norteia esse trabalho é: Quais são as tecnologias de informação aplicadas à logística adotadas pelos agentes de carga internacionais (IFFs) atuantes no Brasil?

O estudo se justifica, pois temas como a integração entre diferentes tipos de aplicação, pesquisa empírica sobre a adoção das TIC e o papel de fornecedores de tecnologia no processo de adoção não estão totalmente representados na literatura (PEREGO; PEROTTI; MANGIARACINA, 2011). Além disso, Marchet, Perego e Perotti (2009) ressaltam que o tema da adoção de tecnologia é explorado no transporte público, mas não no transporte de carga, e a atenção até agora tem sido focada em aplicações específicas ou tecnologias habilitadoras, em vez de fornecer um quadro abrangente para a análise das TIC no âmbito global da indústria de transportes.

Os resultados mostram que as tecnologias SGF, TAG, AFT e TM/TMS são importantes para os IFFs, porem tem adesão baixa evidenciando ao passo que tecnologias com adoção alta já não se mostram tão importantes. Os dois fatos remetem a situações gerenciais não otimizadas e desperdício de recursos

\section{REFERENCIAL TEÓRICO}

Admitindo que "a essência da formulação de uma estratégia competitiva é relacionar uma companhia ao seu meio ambiente" (PORTER, 1986, p.22), é importante o entendimento da atividade logística em si, pois conecta a 
companhia ao seu meio. Tradicionalmente, a logística concentrou-se no fluxo eficiente de bens ao longo do canal de distribuição, conferindo menos importância para o fluxo de informações. Atualmente, essa situação mudou ampliando-se a quantidade e a qualidade das informações, exemplo disso são: informações sobre status do pedido, disponibilidade de produtos e definições precisas de prazos são elementos necessários do serviço total ao cliente. A informação aumenta a flexibilidade permitindo identificar (qual, quanto, como, quando e onde) os recursos que podem ser utilizados para que se obtenha vantagem estratégica. (NAZÁRIO, 1999).

Num sentido amplo, autores (NEGROPONTE, 1995; GATES, 1995) apontam que a mudança de século foi a passagem para uma era pós-informação, caracterizada pela personalização das informações e remoção de barreiras geográficas.. Todavia, é no campo dos negócios que a Internet vem avançando de forma revolucionária, produzindo o que Drucker (1999) chama de mudança radical do significado da informação, ultrapassando o conceito tradicional de utilização da informação operacionalmente, para transformá-la em ferramenta auxiliar à tomada de decisões estratégicas, verdadeira tarefa da alta gerência Logo, a importância da TI para a logística se refletem na competitividade das empresas.

Assim esta seção se divide em duas partes. Na primeira analisa-se a atividade dos Freight Forwarding, na segunda abordam-se as tecnologias possíveis e utilizadas objetivando-se relacioná-las com as atividades de agenciamento e seu impacto.

\section{A ATIVIDADE DE AGENCIAMENTO DE CARGAS (FREIGHT FORWARDING)}

Pela definição do Council of Supply Chain Management Professionals, logística é a parte do gerenciamento da cadeia de abastecimento que planeja, implementa e controla o fluxo e armazenamento eficiente e econômico de matérias-primas, materiais semiacabados e produtos acabados, bem como as informações a eles relativas, desde o ponto de origem até o ponto de consumo, com o propósito de atender às exigências dos clientes (CARVALHO, 2002). A logística tem como função agrupar conjuntamente as atividades relacionadas ao fluxo de produtos e serviços para administrá-las de forma coletiva, podendo ser considerada uma evolução natural do pensamento administrativo e tem o objetivo de providenciar bem e serviços corretos, no lugar certo, no tempo exato e na condição desejada ao menor custo possível (BALLOU, 2001).

Melacini, Marchet e Perotti, (2013) argumentam sobre a importância do transporte de cargas relacionando com a participação no produto interno bruto de um país, e porque impactam praticamente todos os setores da economia, sendo responsável por uma parcela considerável dos custos logísticos (PEREGO; PEROTTI; MANGIARACINA, 2011). Devido a isso as empresas optam pela terceirização do transporte carga como forma de reduzirem os custos agregados, acréscimo de qualidade, ganhos de facilidades fiscais, obtenção de benefícios da gestão de risco ou de especialização operacional (SîRBU; NAG; PINTEA, 2012). Porem Simchi-Levi et al. (2003) lembram que o processo de outsourcing logístico oferece tanto vantagens e desvantagens. A grande desvantagem é a perda de controle de uma função específica pela parte contratante. 
Segundo a International Federation of Freight Forwarders Associations (FIATA), o agenciamento de cargas e a prestação de serviços logísticos são atividades de qualquer natureza relacionadas ao transporte (executado de modo uni ou multimodal), tais como consolidação, armazenagem, manuseio, embalagem ou distribuição de mercadorias e seus serviços secundários, incluindo desembaraço aduaneiro e assuntos fiscais, emissão de certificados para propósitos oficiais, contratação de seguro e obtenção de pagamentos ou documentos relativos à mercadoria. O agenciamento de cargas, ou freight forwarding, também inclui serviços de informação e comunicação conectados ao transporte, manuseio ou armazenagem da carga.

Nesse sentido, freight forwarder, forwarder, forwarding agent, non-vessel operating common carrier (NVOCC), é uma pessoa ou empresa que organiza expedições para indivíduos ou empresas para obter mercadorias do fabricante ou produtor a um mercado, cliente ou ponto final de distribuição (RANDOM HOUSE DICTIONARY, 2001). Esses prestadores de serviço podem usar uma variedade de modos de transporte, incluindo navios, aviões, caminhões e ferrovias, e muitas vezes vários modos para uma única remessa. Os agentes de carga internacionais possuem conhecimentos adicionais na preparação e processamento de qualquer documentação e execução de atividades relativas às transferências internacionais. São os consolidadores de cargas marítimas, empresas que se dedicam ao agrupamento de pequenos lotes de mercadorias de pequenos embarcadores, buscando otimizar a ocupação do espaço de um contêiner (VIEIRA; RODRIGUES, 2005). Pode-se considerar que, enquanto os armadores estão mais voltados ao transporte de contêineres completos, denominados Full Container Loads (FCL), os NVOCCs dedicam-se ao transporte de cargas fracionadas, denominadas consolidadas ou Less than Container Loads (LCL). Do lado da terra, os International Freight Forwarders (IFFs), também chamados no Brasil de agentes internacionais de carga (VIEIRA et al., 2007; VIEIRA; PASA; ARENHART, 2008), apresentam uma atuação mais ampla, uma vez que têm como especialidade prover uma variedade de funções que facilitam a movimentação de embarques internacionais (MURPHY; DALEY, 1997). Tais funções englobam tanto cargas $\mathrm{FCL}$ como $\mathrm{LCL}$ e incluem também serviços aduaneiros e de transporte terrestre, podendo chegar, em alguns casos, a projetos logísticos especiais que cobrem grande parte das necessidades dos clientes.

\section{TECNOLOGIAS DE INFORMAÇÃO APLICADAS À LOGÍSTICA}

A informação sempre foi um elemento importante para as operações logísticas, sem o qual nenhum aspecto da cadeia de suprimentos conseguiria proporcionar um alto nível de desempenho. Dias et al. (2003) enumeram os seguintes benefícios decorrentes do uso da TI na SCM: i) compartilhamento de informações instantâneas; ii) compartilhamento de programas que aumentam a eficiência operacional; iii) acompanhamento em tempo real, pelo consumidor, da carga; e iv) desenvolvimento de canais de venda globais, redução dos estoques e maior flexibilidade. Jagersma (2011) amplia dizendo que impacta a capacidade de inovação e criatividade (através da utilização de informação para o pensamento cruzado para desenvolver novas propostas de clientes e apoiar novas formas de atividade comercial). Assim, os avanços da TI aplicado ao transporte de carga se confundem com o próprio desenvolvimento e evolução do transporte de carga 
(CRAINIC; GENDREAU; POTVIN, 2009). Principalmente no planejamento e na otimização de custos através da redução de papéis e agilidade no fluxo das informações (MARCHET; PEREGO; PEROTTI, 2009).

Segundo Laudon e Laudon (2007), as empresas usam sistemas de gerenciamento da cadeia de suprimentos (SCM) para trocar informações com seus fornecedores sobre disponibilidade de materiais e componentes, datas de entregas para remessas de suprimentos e requisitos de produção. Também usam esses sistemas para trocar informações com distribuidores e expedidores a respeito de níveis de estoque, andamento dos pedidos e datas de entrega para expedição de produtos acabados. Dentre as TIs mais difundidas, se encontram o Electronic Data Interchange (EDI), o Warehouse Management System (WMS), o Vendor Managed Inventor (VMI), Radio-Frequency IDentification (RFID), o Global Positioning System, ou sistema de posicionamento global (GPS), o Transportation Management System (TMS), o Enterprise Resource Planning (ERP).

Para Turban et al. (2004), o EDI pode ser definido como o movimento eletrônico de documentos padrão de negócio entre, ou dentro, de empresas. 0 EDI usa um formato de dados estruturado de recolha automática que permite que os dados sejam transformados sem serem reintroduzidos. 0 principal uso do EDI é transferir transações de negócio repetitivas tais como: encomendas, faturas, aprovações de crédito e notificações de envio (TURBAN et al., 2004).

De acordo com Banzato (1998), um WMS é um sistema de gestão de armazéns, que aperfeiçoa todas as atividades operacionais (fluxo de materiais) e administrativas (fluxo de informações) dentro do processo de armazenagem, incluindo recebimento, inspeção, endereçamento, estocagem, separação, embalagem, carregamento, expedição, emissão de documentos, inventário, entre outras, que integradas atendem às necessidades logísticas, maximizando os recursos e minimizando desperdícios de tempo e de pessoas.

VMI (IGF) é um programa desenvolvido por uma parceria entre fabricante e fornecedor direcionado para a gestão de estoques e controle da informação de ordens de compra/venda, em que se assume como uma mais valia em relação à troca de informação através do Electronic Data Interchange (EDI). O IGF surge como uma forma de lidar com a incerteza da procura, na medida em que coordena os diferentes elos da cadeia de abastecimento no difícil processo de prever a procura (RAGHUNATHAN, 2001).

A identificação por radiofrequência ou RFID é um método de identificação automática através de sinais de rádio, recuperando e armazenando dados remotamente através de dispositivos denominados etiquetas RFID. Assim como o código de barras (e diferentes etiquetas/Tags), é uma forma de identificação eletrônica.

O Sistema de Gerenciamento de Transporte ou ainda Sistema de Gestão de Transporte e Logística (TMS), é um software para melhoria da qualidade e produtividade de todo o processo de distribuição. Este sistema permite controlar toda a operação e gestão de transportes de forma integrada. O sistema é desenvolvido em módulos que podem ser adquiridos pelo cliente, consoante as suas necessidades (GASNIER; BANZATO, 2001)

O Enterprise Resource Planning (ERP) ou planejamento de recurso corporativo é um sistema de informação que integra todos os dados e processos 
de uma organização em um único sistema. A integração pode ser vista sob a perspectiva funcional (sistemas de finanças, contabilidade, recursos humanos, fabricação, marketing, vendas, compras etc) e sob a perspectiva sistêmica (sistema de processamento de transações, sistemas de informações gerenciais, sistemas de apoio à decisão, etc.). O ERP é uma plataforma de software desenvolvida para integrar os diversos departamentos de uma empresa, possibilitando a automação e armazenamento de todas as informações do negócio (TURBAN, 2010).

\section{TECNOLOGIAS DE INFORMAÇÃO UTILIZADAS PELOS FREIGHT FORWARDERS}

A importância da TI tem sido estudada por diversos autores. Davenport e Short, (1990) apontam a importância na melhoria e redesenho do processo logístico como um todo, Já Jagersma (2011) foca na capacidade de gerenciamento logístico impactando a competitividade, e Perego, Perotti e Mangiaracina, (2011) enfocam no transporte de cargas.

Uma referencia importante sobre o assunto trata-se de Marchet, Perego e Perotti (2009), pois consultando o Google Academics observa-se que o artigo é citado por 112 outros papers e na base de dados de artigos científicos Emerald possui 6709 downloads desde 2011. Marchet, Perego e Perotti (2009) classificaram as aplicações de TI disponíveis para empresas de transporte de cargas e logística em quatro tipos: i) aplicações de gerenciamento de transporte ou Transportation Management (TM); ii) aplicações de execução da cadeia de suprimentos ou Supply Chain Execution (SCE); iii) aplicações de automação da força de campo ou Field Force Automation (FFA); e iv) aplicações de gestão de frota e fretes ou Fleet and Freight Management (FFM).

As aplicações de gerenciamento de transporte ou Transportation Management (TM) são ferramentas de apoio à decisão no planejamento, otimização e execução de transporte, com todas as funcionalidades típicas, incluindo agendamento, acompanhamento de embarque e rastreamento, pagamento e auditoria de frete, podendo estar incluso no SCE.

A execução da cadeia de fornecimento (SCE) são aplicações orientadas para a execução, incluindo sistemas de gestão de armazéns (WMS), sistemas de gestão de transportes (TMS), sistemas de gestão de comércio global (GTM) e outras aplicações de execução, tais como sistemas de apoio à decisão em tempo real (roteamento dinâmico, sistemas de fornecimento dinâmico e sistemas de visibilidade da cadeia de suprimentos dentro da empresa) (GARTNER, 2017). 0 SCE é uma evolução do SCM e da logística, pois amplia os conceitos que geram valor aos produtores, aos intermediários e aos clientes. O processo de SCM iniciase no desenvolvimento do produto, inclui o planejamento de marketing, a fabricação, as operações financeiras, as compras e o desenvolvimento de fornecedores (FLEURY, 1999).

As aplicações de automação da força de campo ou por sua vez, são soluções possibilitadas pela tecnologia móvel e de apoio à integração entre a força de trabalho remota e processos de negócios corporativos.

A Field Force Automation (FFA) é composta por funcionários que trabalham fora de sua base de operações durante a execução de seus processos de trabalho (RODINA; ZEIMPEKIS; FOUSKAS, 2003). Devido ao isolamento físico dos 
funcionários da FFA, apresentam-se dificuldades específicas como: coordenação, atribuição de empregos e rastreamento da evolução do trabalho, entre outros (GRUHN; HULDER; IJIOUI, 2003)

Fleet Freight Management (FFM) são ferramentas de relatório que incluem informações de viagem do veículo (tempos de viagem, tempos de serviço, ponto de entrega, tempos de descolagem) e outros parâmetros, tais como temperatura de carga (útil para produtos congelados e frescos) ou segurança de porta (útil para produtos de alto valor) apoiado por tecnologias RFID.

Em suma, a literatura não permite uma aproximação ideal ao tema em estudo (tecnologias de informação aplicadas aos agentes internacionais de carga atuantes no Brasil), mas provê uma estrutura geral que pode ser considerada como ponto de partida para sua análise. Nesse ponto, devem-se considerar as especificidades do setor (logística internacional) e dos próprios prestadores de serviço (IFFs), que possuem necessidades e níveis de adoção de tecnologias de informação distintos em função dos setores a que servem e dos contextos em que estão inseridos.

Outra classificação de tecnologias de informação utilizadas na logística internacional é a de Vieira e Santos (2012). Considerando o seu propósito, os autores classificaram os sistemas de informação utilizados pelos IFFs em quatro tipos: i) transacional; ii) de controle gerencial; iii) de apoio à tomada de decisão; e iv) de planejamento estratégico.

O sistema transacional é a base para as operações logísticas e a fonte para atividades de planejamento e coordenação. Já o sistema de controle gerencial permite que se utilizem as informações disponíveis no sistema transacional para o controle e gerenciamento das atividades logísticas. O sistema de apoio à decisão, por sua vez, caracteriza-se pelo uso de softwares de apoio as atividades operacionais, táticas e estratégicas. E por fim, o sistema de planejamento estratégico das informações logísticas serve como base para o desenvolvimento de estratégias logísticas.

Ao analisar diferentes dimensões da atuação dos IFFs (contratação de frete internacional e nacional, coordenação, consolidação e desconsolidação de cargas, armazenagem e distribuição de mercadoria, assessoria nas questões aduaneiras e fiscais, contratação de seguro, coleta e serviços logísticos) no Estado do Rio Grande do Sul, os autores identificaram os padrões mais recorrentes quanto ao segundo tipo, de controle gerencial. Especificamente no que refere aos sistemas de informação adotados pelos IFFs, confrontando ambas as abordagens (MARCHET; PEREGO; PEROTTI, 2009; VIEIRA; SANTOS, 2012) e considerando o propósito das aplicações de TI, observa-se que, enquanto Marchet, Perego e Perotti (2009) abordam a questão sob um ponto de vista mais operacional, já Vieira e Santos (2012) a analisam sob um ponto de vista mais estratégico. Assim, as abordagens se complementam e se assemelham nas classificações.

\section{PROCEDIMENTOS METODOLÓGICOS}

Objetivando entender o papel das tecnologias da informação no ambiente dos agentes internacionais de carga, foi utilizada a pesquisa descritiva com uma abordagem quantitativa, operacionalizada mediante questionário (survey), normalmente utilizado para pesquisas de opinião pública, pesquisas de mercado 
sobre preferências do consumidor, estudos acadêmicos sobre preconceito, estudos epidemiológicos, entre outros (BABBIE, 2005).

De fato, a pesquisa quantitativa busca evidência conclusiva, baseada em amostras, representativas e, de alguma forma, aplica análise estatística (MALHOTRA, 2006). De acordo com Gil (2008), a pesquisa descritiva visa relatar as características de determinada população ou fenômeno, ou o estabelecimento de relações entre variáveis. Esse tipo de pesquisa envolve o uso de técnicas padronizadas de coleta de dados, tais como o questionário, instrumento utilizado neste estudo (GIL, 2008).

Como procedimentos de coleta de dados seguiu-se o sugerido por Hair Jr. et al. (2005) ao descrever que o processo de amostragem compreende: i) definição da população alvo; ii) seleção da estrutura de amostragem; iii) seleção do método de amostragem; iv) determinação do tamanho da amostra, e v) implementação do plano de amostragem. Assim, a coleta de dados foi realizada de 29/09/2016 a $3 / 11 / 2016$, por meio de um questionário, estruturado em três seções, enviado a uma lista de 233 freight forwarders, obtida através do Guia Marítimo. Utilizou-se o modelo de formulários eletrônicos do sistema Google Docs.

Primeiramente foram enviadas solicitações de resposta por e-mails com o questionário anexado e posteriormente foram executadas ligações telefônicas para sensibilizar sobre a importância do estudo. Retornaram 25 questionários válidos, o que evidencia uma taxa de retorno de 10,7\%, que pode ser considerada alta para pesquisas do tipo survey.

Na primeira seção foram levantadas questões sobre a identificação dos respondentes/empresas. Na segunda seção, foram redigidas questões referentes às tecnologias abordadas pelo estudo (ERP, EDI, TM/TMS, WMS, GPS, Tag's, Gerenciamento de frota, de Automação da Força de Trabalho e SCM), com o objetivo de se compreender o nível de adesão às mesmas. Foi utilizada uma escala de 1 a 5 (da não utilização à adoção total), estruturada da seguinte forma: 1- Não utiliza o sistema; 2- Utiliza raramente o sistema; 3- Utiliza algumas vezes o sistema; 4- Utiliza frequentemente o sistema; 5- Utiliza sempre o sistema. Na terceira seção, seguiu-se a mesma lógica e ordem da seção 2, porém com enfoque na importância atribuída às supracitadas tecnologias ao invés do grau de adesão. A escala, novamente de 1 a 5, estende-se de importância muito baixa (1) a muito alta (5), conforme detalhado a seguir: 1- Importância Muito Baixa; 2Importância Baixa; 3- Importância Média; 4- Importância Alta e 5- Importância Muito Alta.

A análise de dados do estudo foi realizada mediante procedimentos de estatística descritiva, tais como médias, desvios padrão e cruzamento de dados para identificar as semelhanças ou diferenças de percepções (MALHOTRA, 2005). Recebidos os dados finais, foram calculadas as quantidades de respostas para cada elemento da escala e seus respectivos percentuais individuais, considerando-se cada tecnologia e, obtendo-se, assim, cada valor das escalas de Adesão e Importância.

A análise dos dados foi dividida em três etapas: na primeira, foram analisadas as respostas referentes à adesão da tecnologia de forma descritiva; na segunda, foram analisadas as respostas sobre a Importância atribuída às tecnologias e na terceira parte, confrontou-se o grau de adesão com a importância atribuída. Na terceira etapa foram analisados os pontos em que a 
importância é alta, porém a adesão baixa, e o oposto com adesão é alta, entretanto sua importância é baixa, pois demonstram inconstância entre elas. Para tanto foi utilizada uma matriz de Adoção versus Importância, adaptada de Martilla e James (1977).

Para o cálculo dos elementos da Matriz, utilizou-se a seguinte lógica matemática: Dentro da escala de 1 a 5, multiplicou-se o no de respondentes pelo seu respectivo grau atribuído, para então somar e obter assim uma coordenada única para cada tecnologia, conforme apresentado no exemplo a seguir:

$\operatorname{ERP}($ adoção $)=(7$ respostas $\times 1)+(2$ respostas $\times 2)+(6$ respostas $\times 3)+(2$ respostas $\times 4)+(8$ respostas $\times 5)=77$

$\operatorname{ERP}($ importância $)=(4$ respostas $\times 1)+(1$ resposta $\times 2)+(5$ respostas $\times 3)+(6$ respostas $\times 4)+(9$ respostas $\times 5)=90$

Resultando a coordenada única $\operatorname{ERP}(77,90)$.

\section{RESULTADOS}

A análise dos resultados está estruturada em duas partes: i) caracterização dos respondentes; ii) análise das tecnologias de informação adotadas pelos agentes internacionais de carga atuantes no Brasil. A primeira parte dedica-se explorar os dados de perfil dos respondentes objetivando esclarecer suas condições para responderem as questões, bem como suas procedências e outras informações relevantes. Já a segunda parte da análise é subdividida em três seções: i) adoção das tecnologias de informação pelos agentes internacionais de carga; ii) importância atribuída à cada tecnologia; iii) análise da relação entre o nível de adoção das tecnologias e o seu grau de importância.

\section{Caracterização dos respondentes}

A Tabela 1 mostra o perfil dos respondentes e das empresas, destacando-se que foram obtidos 25 questionários válidos.

Tabela 1: Identificação dos respondentes

$\begin{array}{cccc} & & \text { QUANTIDADE } & \% \\ & \text { Ensino superior incompleto } & 13 & 52 \\ \text { Grau de instrução } & \text { Ensino superior completo } & 9 & 36 \\ & \text { Pós-graduação } & 3 & 12 \\ & \text { Administração } & 4 & 16 \\ \text { Área de formação } & \text { Engenharias } & 2 & 8 \\ & \text { Comércio Internacional } & 14 & 56 \\ & \text { Outra } & 5 & 20 \\ \text { Tempo de empresa } & \text { 6 meses } & 4 & 16 \\ & \text { 6 meses - 1 ano } & 6 & 24 \\ & 1 \text { ano - 3 anos } & 3 & 12 \\ & + \text { de 3 anos } & 12 & 48 \\ & \text { Auxiliar } & 1 & 4 \\ \text { Cargo } & \text { Assistente } & 5 & 20 \\ & \text { Analista } & 7 & 28 \\ & \text { Gerente } & 3 & 12 \\ & \text { Diretor } & 6 & 24 \\ & \text { Outro } & 3 & 12 \\ & \text { SP } & 6 & 24\end{array}$




\begin{tabular}{cccc} 
& SC & 1 & 4 \\
& RS & 17 & 68 \\
Outro & 1 & 4 \\
Funcionários & $1-19-$ Micro & 9 & 36 \\
& $20-99-$ Pequena & 8 & 32 \\
& $100-499-$ Média & 3 & 12 \\
Número de & + de 500 - Grande & 5 & 20 \\
contêineres & 501 a 500 & 12 & 48 \\
& 1001 a 5000 & 3 & 12 \\
& 5001 a 10000 & 6 & 24 \\
& Mais de 10000 & 2 & 8 \\
\hline
\end{tabular}

Fonte: Pesquisa de campo (2016)

Pode-se notar pelo perfil dos respondentes (Tabela 1), que o "grau de instrução" dos mesmos é predominantemente de ensino superior variando de "superior incompleto" com 52\%, a pós-graduação, com $12 \%$, denotando um nível de qualificação alto. Este fato é reforçado pela ausência de respostas no nível de ensino médio, tanto o incompleto quanto o completo.

No quesito "área de formação", a predominância é na resposta "Comércio Internacional ou análoga", com 14 respostas e 56\% de percentual. No item "Tempo de trabalho na área" observa-se que 60\% trabalham a mais de 1 ano ou mais, representando um percentual significativo para o tempo de permanência na empresa.

Na questão do cargo houve uma maior distribuição das respostas entre as diferentes alternativas, pois a opção "Auxiliar" foi a menos selecionada, com apenas 1 respondente. Todas as outras tiveram percentuais maiores, exceto "Supervisor/Coordenador", cujo número de respondentes atuantes neste (s) cargo (s) foi nulo.

No que se refere ao item "Estado", identificando onde as empresas se localizam, notou-se uma predominância de Rio Grande do Sul (68\%), seguido por São Paulo (24\%), Santa Catarina (4\%) e, outro, com apenas um respondente.

No quesito "Porte das empresas" ou no de funcionários, os percentuais também foram bem distribuídos, com leve superioridade de respondentes de micro ou pequenas empresas (totalizando 68\%) em comparação com médias e grandes (32\%), naturalmente mais difíceis de contatar.

Por fim, em relação ao número de contêineres movimentados por ano em portos brasileiros, destacam-se $48 \%$ das empresas que marcaram menos de 500 . Em contrapartida, as outras opções acima de 500 totalizaram 52\%, um número também significativo e condizente com os outros dados apresentados referentes aos respondentes.

No geral, a identificação dos respondentes apresentou uma estratificação adequada ao desenvolvimento do estudo, contemplando todos os principais elementos identificadores nas IFFs, para que se pudesse elaborar uma análise apurada das tecnologias de informação. 
A análise dos resultados está estruturada em três seções: i) adoção; ii) importância de cada tecnologia; e iii) análise da adoção versus importância

\section{ADOÇÃO}

Nesta primeira parte foram analisados os dados referentes à adoção de cada tecnologia (Tabela 2), que se refere à adoção da tecnologia (TEC) para cada nível na escala, em quantidade de respondentes e sua respectiva porcentagem no total, bem como a unidade numeral representativa do grau de adesão, a qual foi utilizada para formar a sua coordenada posteriormente na matriz final (Figura 1).

Tabela 2: Adoção da tecnologia

\begin{tabular}{|c|c|c|c|c|c|c|c|c|c|c|c|}
\hline \multirow{3}{*}{ TEC } & \multicolumn{10}{|c|}{ ADOÇÃO } & \multirow[t]{2}{*}{ GRAU } \\
\hline & \multicolumn{2}{|c|}{1} & \multicolumn{2}{|c|}{2} & \multicolumn{2}{|c|}{3} & \multicolumn{2}{|c|}{4} & \multicolumn{2}{|c|}{5} & \\
\hline & Qtd & $\%$ & Qtd & $\%$ & Qtd & $\%$ & Qtd & $\%$ & Qtd & $\%$ & \\
\hline ERP & 7 & 28 & 2 & 8 & 6 & 24 & 2 & 8 & 8 & 32 & 77 \\
\hline EDI & 9 & 36 & 2 & 8 & 9 & 36 & $x$ & $x$ & 5 & 20 & 65 \\
\hline TMS & 7 & 28 & 4 & 16 & 4 & 16 & 5 & 20 & 5 & 20 & 72 \\
\hline WMS & 12 & 48 & 3 & 12 & 3 & 12 & 4 & 16 & 3 & 12 & 58 \\
\hline GPS & 5 & 20 & 1 & 4 & 5 & 20 & 4 & 16 & 10 & 40 & 88 \\
\hline TAG & 7 & 28 & 2 & 8 & 9 & 36 & 5 & 20 & 2 & 8 & 68 \\
\hline SGF & 10 & 40 & 3 & 12 & 4 & 16 & 4 & 16 & 4 & 16 & 64 \\
\hline AFT & 9 & 36 & 4 & 16 & 3 & 12 & 7 & 28 & 2 & 8 & 64 \\
\hline SCM & 10 & 40 & 1 & 4 & 4 & 16 & 7 & 28 & 3 & 12 & 67 \\
\hline
\end{tabular}

Fonte: Pesquisa de campo (2016)

Em termos de adoção, as tecnologias da informação que obtiveram o nível mais alto foram: o GPS, que obteve dez respostas (40\%) com nível de adoção máxima, sendo a tecnologia mais adotada, seguido do ERP com oito respostas (32\%) e em terceiro lugar a tecnologia TMS, com cinco respostas (20\%) atribuindo-lhe máxima adoção, e outras cinco (20\%) respondentes que atribuíram adoção 4 , totalizando $40 \%$.

No que se refere às menos adotadas, tem-se o WMS com $48 \%$ dos respondentes atribuindo-lhe o nível mais baixo de adoção seguido pelo Sistema de Gerenciamento de frota (SGF) e pelo SCM, que tiveram $40 \%$ de adoção.

\section{IMPORTÂNCIA}

A Tabela 3 representa as percepções de importância atribuídas a cada uma das tecnologias investigadas. 
Tabela 3: Importância atribuída às tecnologias

\begin{tabular}{|c|c|c|c|c|c|c|c|c|c|c|c|}
\hline TEC & \multicolumn{10}{|c|}{ IMPORTÂNCIA } & \multirow[t]{2}{*}{ GRAL } \\
\hline 0 & \multicolumn{2}{|c|}{1} & \multicolumn{2}{|c|}{2} & \multicolumn{2}{|c|}{3} & \multicolumn{2}{|c|}{4} & \multicolumn{2}{|c|}{5} & \\
\hline$n$ & Qtd & $\%$ & Qtd & $\%$ & Qtd & $\%$ & Qtd & $\%$ & Qtd & $\%$ & \\
\hline$t$ ERP & 4 & 16 & 1 & 4 & 5 & 20 & 6 & 24 & 9 & 36 & 90 \\
\hline e EDI & 6 & 24 & 3 & 12 & 6 & 24 & 5 & 20 & 5 & 20 & 75 \\
\hline : TMS & 5 & 20 & 2 & 8 & 5 & 20 & 8 & 32 & 5 & 20 & 81 \\
\hline WMS & 6 & 24 & 2 & 8 & 9 & 36 & 5 & 20 & 3 & 12 & 72 \\
\hline p GPS & 4 & 16 & 2 & 8 & 3 & 12 & 3 & 12 & 13 & 52 & 94 \\
\hline $\mathrm{e}^{\mathrm{TAG}}$ & 4 & 16 & 3 & 12 & 7 & 28 & 7 & 28 & 4 & 16 & 79 \\
\hline s SGF & 5 & 20 & 2 & 8 & 6 & 24 & 7 & 28 & 5 & 20 & 80 \\
\hline q AFT & 5 & 20 & 2 & 8 & 8 & 32 & 7 & 28 & 3 & 12 & 76 \\
\hline SCM & 6 & 24 & 2 & 8 & 7 & 28 & 6 & 24 & 4 & 16 & 75 \\
\hline
\end{tabular}

Fonte: Pesquisa de campo (2016)

Assim, de acordo com a Tabela 3, as tecnologias ERP e GPS foram as consideradas mais importantes, com $60 \%$ e $64 \%$, respectivamente, atribuindoIhes níveis 4 e 5 na escala utilizada. Logo em seguida vem os sistemas de TM/TMS, com 52\% de importância alta a muito alta e, então, o sistema de gerenciamento de frota, um pouco abaixo, com $48 \%$ dos respondentes the atribuindo tal importância. A(s) tecnologia(s) de Tags (rastreio eletrônico, etiqueta eletrônica, RFID e semelhantes) utilizada para identificação e automação aparece na sequência com os níveis 4 e 5 de importância atribuída por $44 \%$ dos respondentes.

As tecnologias de informação com pontuações mais baixas em importância foram o EDI com $36 \%$ dos respondentes considerando-o com importância muito baixa a baixa, seguido por WMS e SCM, obtendo $32 \%$ da mesma classificação.

\section{ADOÇÃO VERSUS IMPORTÂNCIA}

Confrontando ambas as análises (adoção Tabela 2 e importância Tabela 3), obteve-se a Matriz apresentada na Figura 1, na qual se observa nos quadrantes superior esquerdo (adesão alta e importância baixa; desperdício de recurso) e inferior direito (importância alta e adesão baixa; desperdício de potencial), os objetos norteadores desse estudo.

Portanto, ao se encontrar tais marcações, pode-se notar uma falha de consistência entre as variáveis consideradas e apresentadas, mostrando os principais pontos de desperdícios que podem afetar a performance e o resultado dos IFFs no cotidiano de suas atividades. Os valores das coordenadas estão representados na Tabela 4, os quais permitem que se possa construir a matriz (Figura 1) facilitando sua visualização e entendimento. 
Tabela 4: Valores dos elementos da matriz de adoção versus importância.

$\begin{array}{ccc}\text { Tecnologia } & \text { Nível de Adoção } & \text { Nível de Importância } \\ \text { ERP } & 77 & 90 \\ \text { EDI } & 65 & 75 \\ \text { TMS } & 72 & 81 \\ \text { WMS } & 58 & 72 \\ \text { GPS } & 88 & 94 \\ \text { TAG } & 68 & 79 \\ \text { SGF } & 64 & 80 \\ \text { AFT } & 64 & 76 \\ \text { SCM } & 67 & 75\end{array}$

Fonte: Pesquisa de campo (2016)

A matriz, apresentada na Figura 1, divide-se em quadrantes. Como as escalas utilizadas possuíam 5 níveis, (de 1 a 5), para definir as linhas de centro da matriz, as quais geraram quatro quadrantes, considerou-se uma distribuição uniforme das respostas, ou seja, cinco respostas para cada ponto da escala, considerando que o total de respostas foi 25 , conforme apresentado a seguir: $5 \times 1+5 \times 2+5 \times 3+$ $5 \times 4+5 \times 5=75$. Portanto os dois eixos da matriz foram divididos medianamente no valor de 75 (setenta e cinco), representando a divisão entre as coordenadas.

Figura 1: Matriz de Adoção versus Importância

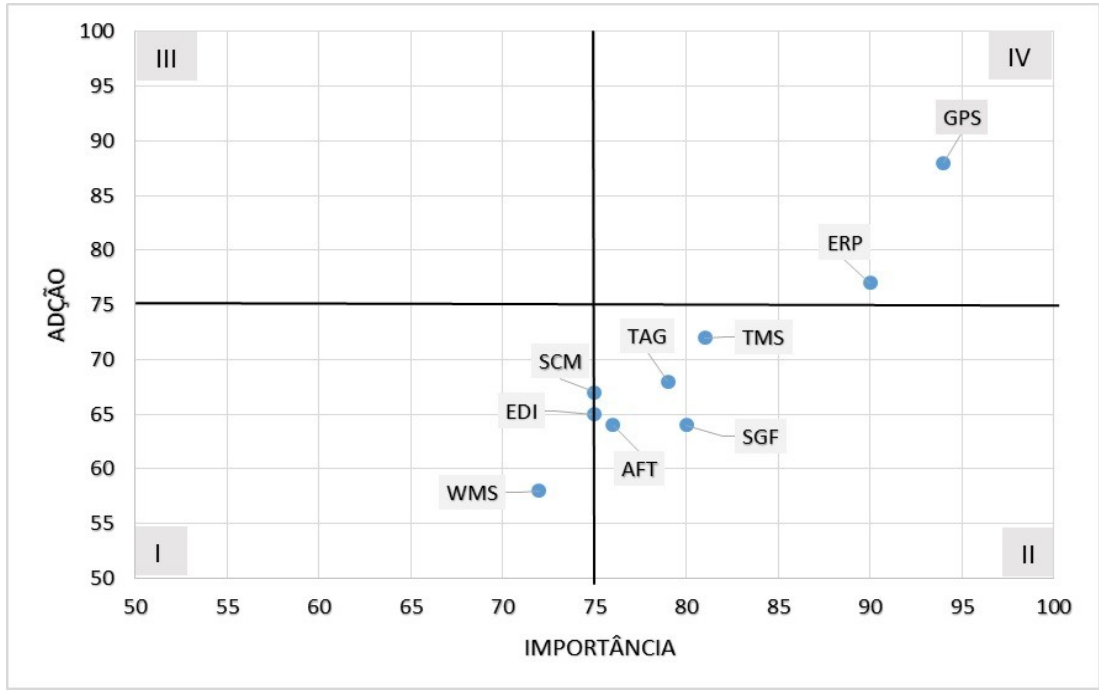

Fonte: Pesquisa de campo (2016)

Analisando-se os quadrantes (I, II, III e IV) na Figura 1, pôde-se verificar o nível de relação entre a adoção e a importância das tecnologias de informação.

O Quadrante I é definido por alto nível de adoção e pouca importância atribuída, não obteve indicação de nenhuma tecnologia. Esse fato permite concluir que, a capacidade dos IFFs questionados de administrar suas tecnologias, ao menos, não está sendo utilizada de modo a desperdiçar recursos, tanto humanos quanto financeiros. Pois, a princípio, adotar intensamente uma tecnologia a qual não se atribui importância mostra um erro gerencial atual ou mesmo proveniente de uma decisão estratégica ruim no passado.

Em seguida tem-se o Quadrante II, o qual apresenta nível de importância e de adesão igualmente baixos. Isso significa que as tecnologias aqui estão em 
equilíbrio funcional. Identificou-se neste quadrante apenas a tecnologia de informação WMS, significando que as IFF não acreditam que o WMS possa contribuir tão intensamente com sua performance quanto outras tecnologias. A gestão dos armazéns não se mostra, portanto, importante. Isso pode ser atribuído ao fato de muitos IFFs não possuírem armazém próprio.

Entre os quadrantes II e III aparecem dispostas exatamente sobre a linha divisora vertical, porém abaixo da linha divisória horizontal, as tecnologias EDI $(65 ; 75)$ e SCM $(67,75)$, ambas com importância média, mas com nível de adoção baixo. $O$ que se pode depreender do fato são duas visões. A primeira é que as duas tecnologias venham a se posicionar, no futuro, no quadrante II (baixo grau de importância), portanto perdendo sua importância média atual, reduzindo-se esforço no seu uso. A segunda é que as tecnologias se posicionem, no futuro, no quadrante III o que pode significar que as mesmas cresceram em termos de importância, mas não apresentaram elevado grau de adoção, este fato exigirá investimentos e esforços para que sua adoção se amplie.

Ainda no quadrante II, numa análise mais apurada sobre o EDI, por ser uma tecnologia madura, deve ser considerada a possibilidade de que o EDI tenha atingido um nível de saturação dentro da cadeia logística, em especial no setor estudado, sendo substituído por opções mais modernas inclusas em outros sistemas integrados. Já o caso da tecnologia de SCM evidencia que os IFFs têm uma atuação autônoma, abrindo mão de uma integração maior, surgindo uma oportunidade para ampliação do relacionamento com seus clientes e fornecedores, a partir da intensificação do seu uso.

Seguindo-se para o Quadrante III, observam-se as tecnologias que são entendidas como importantes, mas com baixo nível de adoção TMS $(72 ; 81)$, TAG $(68,79)$, SGF $(64,80)$, AFT $(64,76)$. São tecnologias que deverão receber maior investimento para que, ampliando sua adoção, seja viável retirar maior proveito de sua importância. Pode-se deduzir também, que estas tecnologias estarão nos planos de investimento dos agentes de carga que não as utilizam de forma arquétipa.

Por fim, no Quadrante IV estão representadas as tecnologias de alta adoção e de alta importância na visão dos respondentes que são, $\operatorname{ERP}(77,90)$ e $\operatorname{GPS}(88$, 94). O ERP pela sua amplitude e abrangência no controle gerencial das operações transacionais diárias tem ocupado destaque nas organizações de um modo geral. Já o GPS vem a atender a demanda pela necessidade de respostas imediatas e controle de tempos no atendimento do transporte.

Assim, pode-se concluir que estas tecnologias de alta importância e que são altamente adotadas estão alinhadas com a estratégia empresarial, tanto sob o aspecto da gestão estratégia quanto da gestão operacional.

\section{DISCUSSÃO DOS RESULTADOS}

O objetivo geral deste estudo foi analisar o papel das tecnologias de informação aplicadas à logística internacional adotadas pelos agentes internacionais de carga (IFFs) atuantes no Brasil. A análise está sintetizada na

Figura 1 que é a matriz resultante do relacionamento da adoção das tecnologias versus sua importância sob o ponto de vista dos respondentes. 
Observa-se, primeiramente, que todas as empresas utilizam mais do que uma das tecnologias objetivando obter benefícios, de acordo com o que Haley e Krishnam (1995) alegam, pois a logística é a área empresarial que mais se beneficiou da automatização e da redução dos custos permitida pela $\mathrm{TI}$, produzindo um melhor uso da informação (DRUCKER, 1990). Cabe ressaltar que o resultado ideal da utilização da TI está intimamente ligado à capacidade e a forma como as pessoas a utilizam (DAVENPORT, 1998), este fato remete a necessidade de desenvolvimento de pessoas que além da habilidade técnica nas funções dos IFFs, necessitam da capacidade de lidar e sentirem-se à vontade com o uso das tecnologias de informação.

Analisando-se a Figura 1, no Quadrante IV observa-se que as tecnologias GPS e ERP tem elevado nível de adoção e de importância, mostrando o uso adequado destas tecnologias, pois ao adotarem intensamente tecnologias percebidas como importantes se caracteriza um alinhamento competentes dos IFFs. São tecnologias que têm em suas funções a capacidade de integração, fato fundamental para o transporte de cargas e sua total integração aos processos de logística (BRANSKI; LAURINDO, 2013). Isso facilita o controle e a gestão de um dos elos da cadeia de suprimentos, responsável pelo deslocamento de cargas (CRAINIC; LAPORTE, 1997). O GPS, enquanto permite o reconhecimento em tempo real da posição dos veículos de transporte (terra, mar e ar), facilita a movimentação de matérias-primas e/ou produtos acabados, influenciando positivamente o elemento que representa a maior parcela dos custos logísticos na maioria das empresas (MARCHET; PEREGO; PEROTTI, 2009; PEREGO; PEROTTI; MANGIARACINA, 2011).

A tecnologia menos adotada e com menor nível de importância é o EDI, utilizado para transferir informações em transações de negócio repetitivas, tais como: encomendas, faturas, aprovações de crédito e notificações de envio (TURBAN et al., 2004). Isso se deve ao fato de que o EDI é uma tecnologia madura e que tem suas funções, por vezes, atendidas por outras tecnologias como o ERP.

A tecnologia do SCM, responsável, principalmente, pelo compartilhamento de informações, programas, movimentação e flexibilidade de cadeias de fornecedores/suprimentos (DIAS et al., 2003), tem uma adoção e utilização média (coordenada 67, 75). Embora o potencial de benefício seja interessante o seu uso é difícil na medida em que necessita predisposição em compartilhamento de informações, por vezes tidas como sigilosas este fato limita seu uso.

Nota-se que existe um espaço para crescimento e melhoria no uso de algumas tecnologias (TMS, TAG, SGF e AFT), de tal forma que a integração e o compartilhamento permitido por estes sistemas se tornem mais abrangentes e efetivos. Além disso, a tecnologia de informação SCM não está sendo adotada com intensidade, portanto os IFFs não estão fazendo uso do seu potencial de integração e relacionamento.

No entanto, de um modo geral, conclui-se que as adoções da tecnologia de informação pelos IFFs fazem parte da estratégia organizacional e são percebidas como importantes, este fato mostra que o processo de adoção tende a se ampliar na mesma medida em que seu uso melhora continuamente. 
O estudo objetivou analisar as tecnologias adotadas pelos agenciadores de carga atuantes no Brasil e a importância atribuída para as mesmas. Para tanto, após a coleta e análise dos dados, foi exequível a construção de uma ferramenta que pudesse confrontar, de forma congruente, cada tecnologia entre si e, então, todas no mesmo âmbito.

Resultante desse processo, equiparado com estudos prévios e referenciais teóricos, encontraram-se as tecnologias onde se procurava uma incoerência entre as premissas Adoção e Importância. Essas tecnologias, SGF, TAG, AFT e TM/TMS, cuja presença no quadrante de importância alta e adesão baixa, evidenciaram as áreas que os IFFs não estão otimizando seus recursos e capacidade, pressuposto considerado de difícil percepção no que se refere à gestão de empresas/negócios, especialmente em comparação com o quadrante de adoção alta e importância baixa, o qual concerne um desperdício de recursos, e que não teve nenhuma tecnologia enquadrada na sua classificação.

\section{LIMITAÇÕES DO ESTUDO E SUGESTÕES PARA ESTUDOS FUTUROS}

O estudo realizado, embora tenha sido abrangente, possui algumas limitações no seu desenvolvimento. A primeira refere-se à população e à amostra. Embora a população pesquisada tenha sido de 233 IFFs e se tenha obtido $10,7 \%$ de retorno, a estratificação foi prejudicada pela ausência de respostas de todos os estados. Outra limitação refere-se ao nível hierárquico dos respondentes, que foi variado e que pode significar uma variação no conhecimento dos respondentes a respeito das tecnologias utilizadas.

Como sugestões de estudos futuros, podem-se aprofundar o estudo considerando dois aspectos: o primeiro refere-se a avançar nos estudos qualitativos sobre a intensidade da adoção e da importância das tecnologias, investigando os porquês da não adoção, bem como das tendências tecnológicas para os próximos anos. O segundo aspecto refere-se às empresas de tecnologia, pois aprofundando as razões pelas quais os IFFs dão pouca importância a algumas tecnologias (EDI, WMS e SCM) poderiam ser conduzidas ações para ampliar seus usos e aplicações.

\section{IMPLICAÇÕES GERENCIAIS}

Do ponto de vista das empresas IFFs, as tecnologias de ERP e GPS deveriam ser implementadas pelas empresas que não o fazem, em total capacidade, pois representam a possibilidade de melhor performance com redução de custos.

Do ponto de vistas das empresas de tecnologia, as mesmas deveriam aprofundar o entendimento das razões pelas quais os IFFs dão pouca importância a algumas tecnologias (EDI, WMS e SCM) e se poderiam conduzir ações para ampliar seus usos e aplicações, potencializando suas vendas.

As tecnologias TAG, TMS SGF E AFT são percebidas como importantes, mas a adoção não é alta, então as IFFs deveriam ampliar seu uso objetivando alcançar melhores performance e resultados. No mesmo sentido, as fornecedoras de tecnologia devem demonstrar melhor os benefícios na medida em que devem intensificar a oferta destes produtos as IFFs. 


\title{
Information technologies applied to international logistics
}

\begin{abstract}
Information technology (IT) plays an important role in the development of logistics operations and operator performance. The aims of this research was to analyze the role of information technologies applied to international logistics adopted by cargo agents (IFFs) operating in Brazil. For that, a quantitative research was developed, composed of a structured questionnaire, sent to a sample of IFFs operating in the Brazilian market, with different strategic positions and in different sectors. The results demonstrate the ITs more and less adopted and considered more and less important and, then, the relation between these categories and the medium they are present in.
\end{abstract}

KEYWORDS: Logistics. Information Technology. International Freight Forwarding. IFFs. 


\section{REFERÊNCIAS}

AVILA, G. Estamos na quinta fase da logística? Características e paradigmas da gestão total da cadeia de negócios. Anais eletrônicos: SIMPOI 2006 - FGV - EASP

BABBIE, E. Métodos de Pesquisas em Survey. Belo Horizonte, MG: Editora UFMG. 2005.

BALLOU, R. H. (2001). Gerenciamento da Cadeia de Suprimentos. 4ª edição.

BANZATO, E. WMS - Warehouse management system: Sistema de gerenciamento de armazéns. São Paulo: IMAN, 1998.

BAUER, M. W.; GASKELL, G. Pesquisa qualitativa com texto, imagem e som: um manual prático. Petrópolis: Vozes, 2002.

BRANSKI, R. M.; LAURINDO, F. J. B. (2013). Tecnologia da Informação e integração das redes logísticas. Revista Gestão da Produção, v. 20, n. 2, 2013. crossref

CARVALHO, J. M. C. Logística. 3ª ed. Lisboa: Edições Silabo, 2002.

CRAINIC, T. G. ; GENDREAU, M. ; POTVIN, J. Intelligent freight-transportation systems: Assessment and the contribution of operations research.

Transportation Research Part C: Emerging Technologies, v. 17, n. 6, 2009. crossref

CRAINIC, T. G.; LAPORTE, G. Planning models for freight transportation. European Journal of Operational Research, v. 97, n. 3, p. 409-438, 1997. crossref

DAVENPORT, T. H. Ecologia da Informação: por que só a informação não basta para o sucesso na era da informação. São Paulo: Futura, 1998. 316 pp.

DAVENPORT, T., SHORT, J. E. The new industrial engineering: information technology and business process redesign. Sloan Management Review, v. 31, n. 4, 1990.

DIAS, R.; PITASSI, C.; JOIA, L. Gestão integrada da cadeia de suprimentos. Rio de Janeiro: FGV, EBAPE, 2003.

DRUCKER, P. F. Administrando em Tempos de Grandes Mudanças. São Paulo: Pioneira, 1999. 
FERREIRA, K. A.; ALVES, M. R. P. A. Logística e troca eletrônica de informação em empresas automobilísticas e alimentícias. Revista Produção, v. 15, n. 3, p. 434447,2005 . crossref

FLEURY, P. F. Supply chain management: conceitos, oportunidades e desafios da implementação. In FLEURY, P. F.; WANKE, P.;HARLAND, C. M.; LAMMING, R. C.;

COUSINS, P. D. Developing the concept of supply strategy. International Journal of Operations \& Production Management, v. 19, n. 7, p. 650-673, 1999. crossref

GARTNER, INC. (NYSE: IT). Consultoria e pesquisa. Disponível em: http://www.gartner.com/it-glossary/sce-supply-chain-execution Acesso em: 18 de abril 2017.

GASNIER, D.; BANZATO, E. Armazém Inteligente, Revista LOG Movimentação e Armazenagem, São Paulo, n. 128, Junho, 2001.

GATES, B. A estrada do futuro. São Paulo: Cia. das Letras, 1995.

GIANNOPOULOS, G. The application of information and communication technologies in transport. European Journal of Operational Research, v. 152, p. 302-320, 2004. crossref

GIL, A. C. Como elaborar projetos de pesquisa. 4. ed. São Paulo: Atlas, 2008.

GRUHN, V.; HULDER, M.;IJIOUI, R. Mobile communication systems for truckage companies. Proceedings of the Second International Conference on Mobile Business, Vienna, Austria. 2003.

HAIR JR, J. F. et al. Fundamentos de métodos de pesquisa em administração. Porto Alegre: Bookman, 2005.

HALEY, G.; KRISHNAN, T. It is time for CALM: computer aided logistics management. International journal of physical distribution \& logistics management, v.25, n.4, 1995. crossref

HAMMANT, J. Information technology trends in logistics, Logistics Information Management, v.8, n.6, p. 32-37, 1995. crossref

JAGERSMA P. K. Competitive information logistics. Business Strategy Series, v. 12, n.3, p. $136-145,2011$. crossref 
LAUDON, K.; LAUDON, J. Sistemas de informações gerenciais. São Paulo:

Pearson, 2007.

MALHOTRA, N. K., Introdução à Pesquisa de Marketing. São Paulo: Prentice Halll do Brasil. 2005.

MARCHET, G.; PEREGO, A.; PEROTTI, S. An exploratory study of ICT, adoption in the Italian freight transportation industry. International Journal of Physical Distribution \& Logistics Management, v. 39, n. 9, 2009. crossref

MELACINI, M.; MARCHET, G.; PEROTTI, S. An exploratory study of TMS adoption in the 3PL industry. Global Journal on Technology. v. 3, p. 1390-1399, 2013.

MORESI, D. A. E. Delineando o valor do sistema de informação de uma organização. In: Ciência da Informação. Ci. Inf., Brasília, v. 29, n. 1, p. 14-24, 2000. crossref

MURPHY, P. R.; DALEY, J. M.; HALL, P. K. Carrier selection: do shippers and carriers agree, or not? Transportation Research Part E: Logistics and Transportation Review, v. 33, n. 1, 1997. crossref

MARTILLA, J. A., JAMES, J. C. Importance-performance analysis. Journal of Marketing, n.9, p.41-77, 1977. crossref

NAZÁRIO, P. A importância de sistemas de informação para a competitividade logística. Rio de Janeiro: Centro de Estudos em Logística, Coppead, 1999.

NEGROPONTE, N. A vida digital. São Paulo: Companhia das Letras, 1995.

PASTORE, A. C. et al. Brasil globalizado: o Brasil em um mundo surpreendente. Rio de Janeiro: [S.n.], 2008.

PEREGO, A.; PEROTTI, S.; MANGIARACINA, R. ICT for logistics and freight transportation: a literature review and research agenda. International Journal of Physical Distribution \& Logistics Management, v.41, n. 5, p. 457-483, 2011. doi:10.1108/09600031111138826. crossref

PORTER, M. Estratégia Competitiva: técnicas para análise de indústrias e da concorrência. Rio de Janeiro: Campus, 1986. 
PORTER, M. Vantagem Competitiva - Criando e Sustentando um Desempenho Superior. 17a ed. Rio de Janeiro: Campus, 1989.

RAGHUNATHAN, S.; YEH, A B. (2001). "Beyond EDI: impact of continuous replenishment program (CRP) between a manufacturer and its retailers". Information Systems Research v. 12, p.406-419. 2001. cross ref

RANDOM HOUSE WEBSTER'S UNABRIDGED DICTIONARY. New York: Random House Reference. 2001.

RODINA, E.; ZEIMPEKIS, V.; FOUSKAS, K. Remote workforce business process integration through real-time mobile communications. Proceedings of the Second International Conference on Mobile Business, Vienna, Austria. 2003.

SIMCHI-LEVI, D.; KAMINSKY, P.; SIMCHI-LEVI, E. Cadeia de Suprimentos: Projetos e Gestão. Porto Alegre, Bookman, 2003.

SîRBU, J; NAGY, A; PINTEA, F. R. The Development of Leadership and Business Process Outsourcing (BPO) Skills. Procedia Economics and Finance, Târgu-muresRomênia, v. 3, n.12, p.1141-1147, 2012. DOI: 10.1016/S2212-5671(12)00287-0. crossref

TURBAN, E. Tecnologia da Informação para Gestão: Em Busca de um Melhor Desempenho Estratégico pila grande e Operacional 7ạ ed. ed. (Porto Alegre: Bookman). p. 318-361, 2010.

TURBAN, E.; MCLEAN, E.; WETHERBE, J. Tecnologia da Informação para Gestão: Transformando os Negócios na Economia Digital. Porto Alegre: Bookman, 2004.

VIEIRA, A. Importação práticas, rotinas e procedimentos. 2. ed. Rio de Janeiro: Aduaneiras, 2006.

VIEIRA, G. B. B.; PASA, G. S.; ARENHART, E. J. A atuação de um international freight forwarder pela ótica dos usuários. In: ENCONTRO NACIONAL DE ENGENHARIA DE PRODUÇÃO, 28. 2008, Rio de Janeiro. Anais Eletrônico. Rio de Janeiro: Enegep, 2008. p. 1 - 9. Acesso em: 20 maio 2016.

VIEIRA, G. B. B.; RODRIGUES, S. L. A. O nível de serviço de um consolidador de cargas marítimas sob a ótica dos usuários. Produção Online: Revista Científica Eletrônica de engenharia de Produção, Florianópolis, v. 5, n. 2, p.1-21, jun. 2005. 
VIEIRA, G. B. B; SANTOS, C. H. S. O posicionamento competitivo dos international freight forwarders pela ótica dos exportadores gaúchos, Universidade Tecnológica Federal do Paraná - UTFPR: Revista Gestão Industrial, v.8, n.3, p. 206.

Recebido: 17 nov. 2017

Aprovado: 23 mai. 2018

DOI: $10.3895 /$ gi.v14n3.7352

Como citar:

GONÇALVES, F. S.; VIEIRA, G. B. B.; GONÇALVES, R. B. Tecnologias de informação aplicadas à

logística internacional. R. Gest. Industr., Ponta Grossa, v. 14, n. 3: p. 129-150, 2018, jul./set. 2018.

Disponível em: $<$ https://periodicos.utfpr.edu.br/rgi> . Acesso em: XXX.

Correspondência:

Frederico Schwartzhaupt Gonçalves

Rua Domingos Vanoni, n. 56, AP. 701, Bairro Madureira, Caxias do Sul - RS - Brasil.

Direito autoral: Este artigo está licenciado sob os termos da Licença Creative Commons-Atribuição 4.0

Internacional.

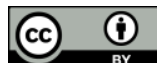

